\title{
CORRECTION
}

\section{Correction: The application of CRISPR/Cas9 system in cervical carcinogenesis}

Chun Gao $\mathbb{D}$, Ping Wu, Lan Yu, Liting Liu, Hong Liu (D), Xiangyu Tan, Liming Wang, Xiaoyuan Huang (D) and Hui Wang (D)

(c) The Author(s), under exclusive licence to Springer Nature America, Inc. 2021

Cancer Gene Therapy (2022) 29:625-626; https://doi.org/10.1038/s41417-021-00393-7

Correction to: Cancer Gene Therapy https://doi.org/10.1038/ s41417-021-00366-w, published online 04 August 2021

There were inaccuracies in Fig. $1 A / B$ and Fig. 3D in the previous version of the article.

In the process of preparing Fig. $1 \mathrm{~A} / \mathrm{B}$, the incorrect representative images were inadvertently into the panel.

In regards to Fig. 3D, when we carefully checked the original data, we found that the name of original data of one part of this figure is not clear enough. Therefore, we chose another picture of serial section of the same group to replace.

We checked in the original data and updated the relevant Figures. We sincerely apologize for any inconvenience or confusion to the readers. The correction made to the Figures does not affect the scientific conclusions of the research.

The original article has been corrected.

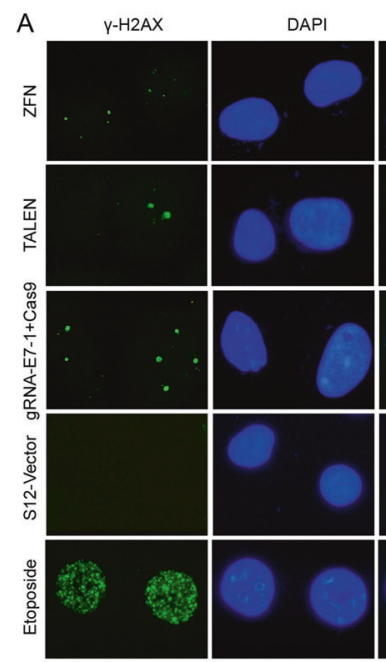

E

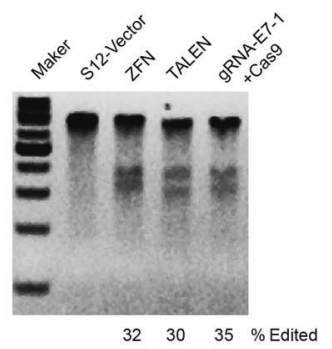

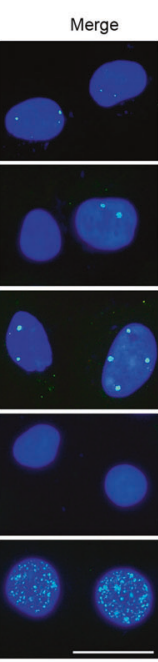

$\mathrm{F}$

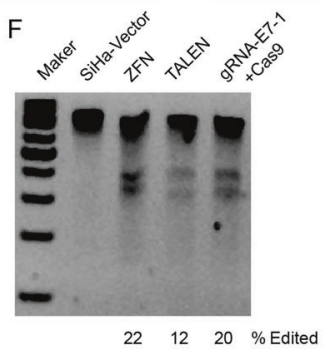

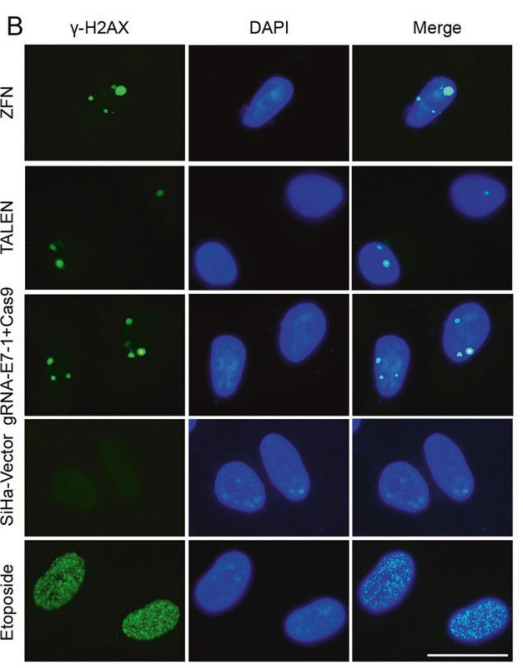
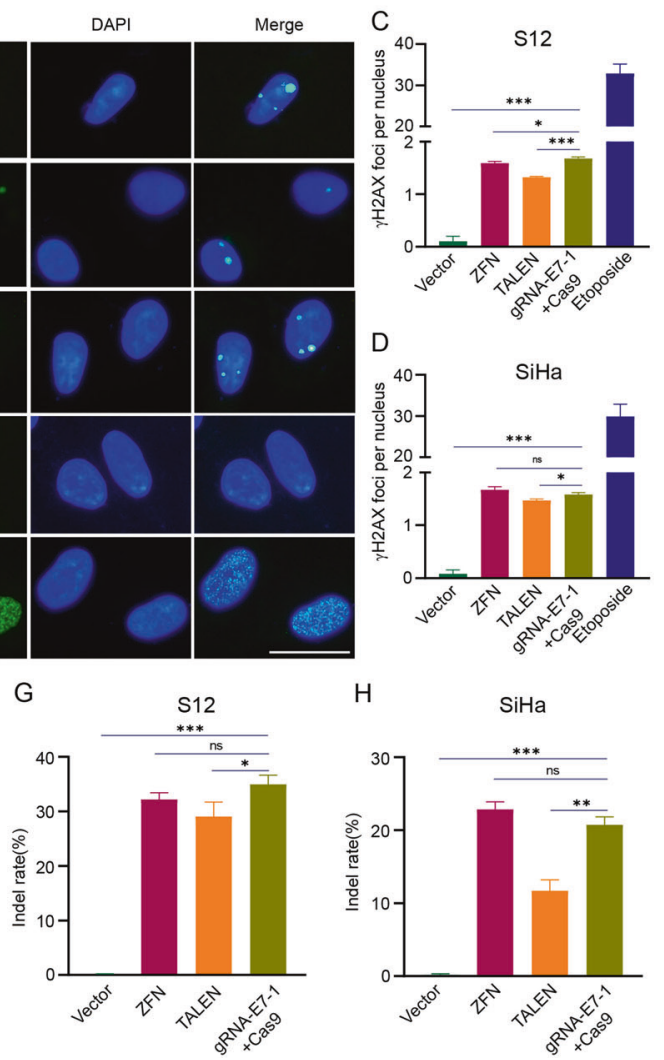

Fig. 1 


\section{Gao et al.}

\section{6}

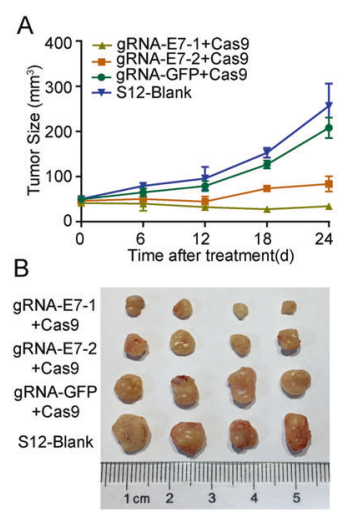

$$
\text { D }
$$

gRNA-E7-1+Ca

gRNA-E7-2+Cas9

GRNA-GFP+Cas 9

S12-Blank

E

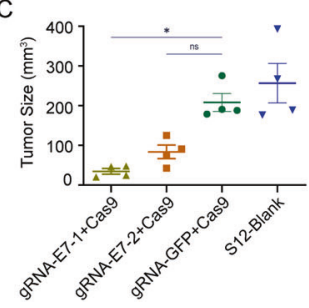

อั
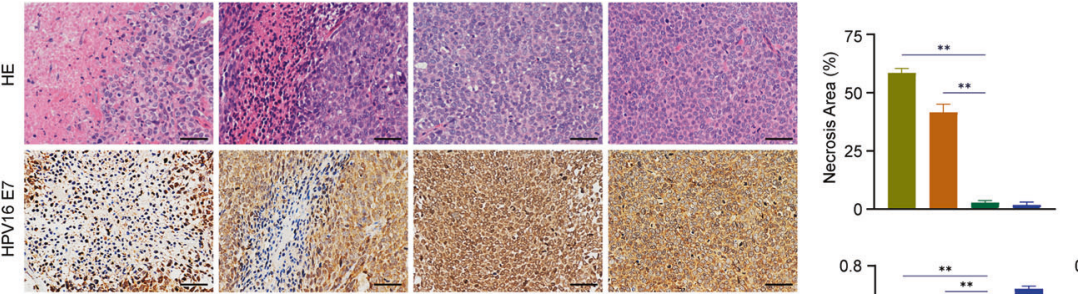

gRNA-E7-1+Cas 9

- gRNA-E7-2+Cas 9

gRNA-GFP+Cas

- S12-Blank

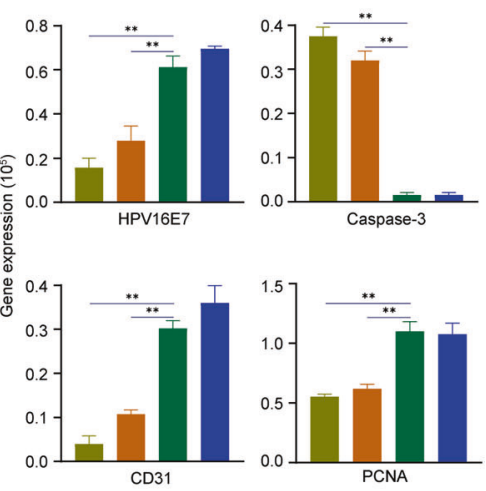

Fig. 3 